\title{
One-relator Kähler groups
}

\author{
INDRANIL BISWAS
}

MAHAN MJ

We prove that a one-relator group $G$ is Kähler if and only if either $G$ is finite cyclic or $G$ is isomorphic to the fundamental group of a compact orbifold Riemann surface of genus $g>0$ with at most one cone point of order $n$ :

$$
\left\langle a_{1}, b_{1}, \ldots, a_{g}, b_{g} \mid\left(\prod_{i=1}^{g}\left[a_{i}, b_{i}\right]\right)^{n}\right\rangle .
$$

32Q15, 57M05, 57M50; 14F35, 32J15

\section{Introduction}

Fundamental groups of compact Kähler manifolds, or Kähler groups for short, have attracted much attention (see Amorós, Burger, Corlette, Kotschick and Toledo [2] for a survey of results and techniques). From a very different point of view, one-relator groups have been studied for a long time in combinatorial group theory (see Lyndon and Schupp [22, Chapter 2]). (A one-relator group is the quotient of a free group with finitely many generators by one relation.) It is natural to ask which groups occur in the intersection of these two classes. In fact one-relator groups have appeared as test cases for various restrictions developed for Kähler groups. Specific examples have been ruled out by Arapura [3, Section 7J]. Restrictions have been obtained from the point of view of rational homotopy theory (see Amorós [1, Sections 3 and 4] and [2, page 39, Examples 3.26 and 3.27]). Further restrictions follow from works of Gromov [18] and Green and Lazarsfeld [16].

In [3, page 12, Section J], Arapura asks which one-relator groups are Kähler. This question was also raised by Amorós. Our aim here is to give a complete answer to Arapura's question. We prove the following (see Theorem 5.5 and Section 5.3):

Theorem 1.1 Let $G$ be an infinite one-relator group. Then $G$ is Kähler if and only if it is isomorphic to

$$
\left\langle a_{1}, b_{1}, \ldots, a_{g}, b_{g} \mid\left(\prod_{i=1}^{g}\left[a_{i}, b_{i}\right]\right)^{n}\right\rangle,
$$

where $g$ and $n$ are some positive integers. 
We show that each of the groups

$$
\left\langle a_{1}, b_{1}, \ldots, a_{g}, b_{g} \mid\left(\prod_{i=1}^{g}\left[a_{i}, b_{i}\right]\right)^{n}\right\rangle, \quad g, n>0,
$$

can in fact be realized as the fundamental group of a smooth complex projective variety. It is known that every finite group is the fundamental group of some smooth complex projective variety (see [2, page 6, Example 1.11]) and so all finite groups are Kähler. Since the only finite one-relator groups are finite cyclic groups, it follows that finite one-relator Kähler groups are precisely the finite cyclic groups.

Therefore, Theorem 1.1 has the following corollary:

Corollary 1.2 Any torsion-free one-relator Kähler group is the fundamental group of some closed orientable surface.

We also prove the following closely related result (see Corollary 4.7):

Theorem 1.3 Let $G$ be a Kähler group such that

- it is a coherent group of rational cohomological dimension two, and

- the virtual first Betti number of $G$ is positive.

Then $G$ is virtually a surface group.

We give an overview of the basic strategy of the proof:

It follows from the structure theory of one-relator groups that they are described as iterated HNN extensions. The Kähler group $G$ we are interested in therefore acts on the Bass-Serre tree $T$ associated to the HNN splitting. If $T$ is not quasi-isometric to the real line, it must be nonamenable and have infinitely many ends. It follows from a refinement (Proposition 2.5) of the theory of stable cuts of Delzant-Gromov [11] using further structure of one-relator groups that $G$ is virtually a surface group in this case.

In case $T$ is quasi-isometric to the real line, then $G$ must be the mapping torus of a free group. These groups are known to be coherent [12]. A simple cohomological dimension argument along with the structure of finitely presented normal subgroups of cohomological dimension 2 groups completes the proof in this case.

The torsion in $G$ is finally handled by further structure theory of one-relator groups. 


\section{Preliminaries}

The reader is referred to Chapter VIII.10 of [7] for generalities on duality and Poincaré duality groups.

Definition 2.1 A group $G$ is a Poincaré duality group of dimension $n$ if $G$ is of type $F P$, and

$$
H^{i}(G, \mathbb{Z} G)= \begin{cases}0 & \text { for } i \neq n \\ \mathbb{Z} & \text { for } i=n\end{cases}
$$

A group $G$ is a duality group of dimension $n$ if $G$ is of type $F P$,

$$
H^{i}(G, \mathbb{Z} G)=0 \quad \text { for } i \neq n,
$$

and $H^{n}(G, \mathbb{Z} G)$ is a nonzero torsion-free abelian group.

Two facts about such groups that we will need are given below; cohomological dimension is denoted by cd.

Theorem 2.2 [5, Theorem 3.5] Let $1 \rightarrow N \rightarrow G \rightarrow Q \rightarrow 1$ be an exact sequence of duality groups. Then $\operatorname{cd}(G)=\operatorname{cd}(Q)+\operatorname{cd}(N)$.

We shall occasionally refer to a group of cohomological dimension $n$ as an $n$-dimensional group.

Theorem 2.3 [4, Theorem B] Let $G$ be a two-dimensional group, and let $H \subset G$ be a finitely presented normal subgroup of infinite index. Then $H$ is free.

The reader is referred to [11] for the notion of cuts, particularly stable cuts, in Kähler groups.

Definition 2.4 Let $Q$ be a group and $R$ a subgroup of it with the following property: If $M$ is a Riemannian manifold with fundamental group $Q$ and $M^{\prime}$ is the cover of $M$ corresponding to $R$, then $M^{\prime}$ has at least three ends and is a nonamenable metric space. We then say that $R$ is a cut subgroup of $Q$.

A group $G$ is said to be virtually a surface group if some finite index subgroup of $G$ is the fundamental group of a closed surface of positive first Betti number.

We shall need the following: 
Proposition 2.5 [6] Let $G$ be a Kähler group fitting in a short exact sequence

$$
1 \longrightarrow N \longrightarrow G \longrightarrow Q \longrightarrow 1,
$$

where $N$ is finitely generated, and one of the following holds:

- $Q$ admits a discrete faithful nonelementary minimal action on a simplicial tree with more than two ends.

- $Q$ admits a (strong-stable) cut $R$ such that the intersection of all conjugates of $R$ is trivial.

Then $G$ is virtually a surface group.

\section{One-relator groups}

All one-relator groups will be assumed to be infinite and the generating set will be assumed to be finite of cardinality greater than one. Our starting point is the following lemma due to Arapura.

Lemma 3.1 [3, Section 7J] Suppose that $G=\left\langle x_{1}, x_{2}, \ldots, x_{n} \mid w\left(x_{1}, \ldots, x_{n}\right)\right\rangle$ is a one-relator Kähler group with $n \geq 2$. Then the following statements hold:

(1) $n$ is even.

(2) Each $x_{i}$ occurs at least once in the word $w$, and the number of occurrences of $x_{i}$ and $x_{i}^{-1}$ coincide.

(3) $G$ surjects onto $\Gamma_{g}$ with $g=n / 2$, where $\Gamma_{g}$ is the fundamental group of a closed Riemann surface of genus $g$.

Remark 3.2 Arapura proves Lemma 3.1 under the assumption that $n$ is strictly greater than two. However, if $n=2$, then it follows that the first Betti number of $G$ satisfies $1 \leq b_{1}(G) \leq 2$ and $b_{1}(G)=2$ if and only if the number of occurrences of $x_{i}$ and $x_{i}^{-1}$ in $w$ coincide for $i=1,2$. Since $G$ is Kähler, we know that $b_{1}(G)$ is even, and hence $b_{1}(G)=2$. Further, $G$ cannot split as a nontrivial free product [18]. Hence each $x_{i}$ occurs at least once in the word $w$ for $i=1,2$. Also, since $b_{1}(G)=2$, the group $G$ surjects onto $\Gamma_{1}$.

We shall now recall some basic structure theory of one-relator groups due to Magnus and Moldavansky. A subgroup $K$ of a one-relator group $G$ is called a Magnus subgroup if it is freely generated by a subset of the generating set of $G$ that omits one of the 
generators present in the defining relator of $G$. If $G$ is an HNN extension of $H$, and $t$ is the free letter conjugating subgroup $A \subset H$ to subgroup $B \subset H$ so that

$$
G=\left\langle H, t \mid A^{t}=B\right\rangle,
$$

then $A$ and $B$ are called the associated subgroups of the HNN extension.

Theorem 3.3 [24, page 250; 25] Let $G=\left\langle x_{1}, \ldots, x_{n} \mid w\right\rangle$ be a one-relator group, where the exponent sum of $x_{1}$ in $w$ is zero. Then the following hold:

(1) There exists a finitely generated one-relator group $H$ whose defining relator has shorter length than $w$.

(2) $G$ is an $H N N$ extension $G=H_{* F}$, where $F$ is a nontrivial free group.

(3) The associated subgroups are Magnus subgroups of $H$.

Finally we shall need two results due to Collins describing intersections of Magnus subgroups.

Theorem 3.4 [10, Theorem 1] Let $G=\left\langle x_{1}, \ldots, x_{n} \mid w\right\rangle$ be a one-relator group, where $w$ is cyclically reduced. Let $M=F(S)$ and $N=F(T)$ be Magnus subgroups of $G$, where $S$ and $T$ are subsets of the generating set, allowing $M=N$. If $M \cap N$ is distinct from $F(S \cap T)$, then $M \cap N$ is the free product of $F(S \cap T)$ and an infinite cyclic group.

Theorem 3.5 [10, Theorem 2] Let $G, M$ and $N$ be as in Theorem 3.4. For any $g \in G$, either $g \mathrm{Mg}^{-1} \cap N$ is cyclic (possibly trivial) or $g \in N M$.

Corollary 3.6 Let $G, S, M$ and $N$ be as in Theorem 3.4. If $S$ has more than one element, and $G \neq N M$, then there exist

$$
g_{1}, g_{2}, g_{3} \in G
$$

such that $g_{1} M g_{1}^{-1} \cap g_{2} M g_{2}^{-1} \cap N \cap g_{3} N g_{3}^{-1}$ is trivial.

Proof By Theorem 3.5, there exists $g=g_{1} \in G$ such that $g_{1} M g_{1}^{-1} \cap N \subset N$ is cyclic. Let $g_{1} M g_{1}^{-1} \cap N=\langle h\rangle$. Since $S$ has more than one element, it follows that $T$ also has more than one element. Hence $N$ is a free group on more than one generator. In particular, there exists $g_{3} \in N$ such that $g_{3}\langle h\rangle g_{3}^{-1} \cap\langle h\rangle=\{1\}$. The proof is completed by setting $g_{2}=g_{3} g_{1}$.

A subgroup $H$ of a group $G$ is said to have height at most $n-1$ if for any $n$ elements $g_{1}, \ldots, g_{n}$ satisfying $H g_{i} \neq H g_{j}$ whenever $i \neq j$, the intersection $\bigcap_{i=1}^{n} g_{i} H g_{i}^{-1}$ is trivial. 
Proposition 3.7 Let $G=\left\langle x_{1}, \ldots, x_{n} \mid w\right\rangle$ be a one-relator group, where $w$ is cyclically reduced. Let $M=F(S)$ and $N=F(T)$ be Magnus subgroups of $G$, where $M \neq N$. Then there exist elements $\left\{g_{i}\right\}_{i=1}^{k},\left\{h_{i}\right\}_{i=1}^{k} \in G^{k}$, for some $k>1$, such that $\left(\bigcap_{i=1}^{k} g_{i} M g_{i}^{-1}\right) \cap\left(\bigcap_{i=1}^{k} h_{i} N h_{i}^{-1}\right)$ is trivial.

Proof By Theorem 3.4, the intersection $M \cap N$ is a finitely generated subgroup of both $M$ and $N$. As $M \neq N$, we may assume without loss of generality that $M \cap N$ is an infinite index subgroup of $M$. Since any finitely generated infinite index subgroup of a free group has finite height [15], the proposition follows.

Proposition 3.8 Let $G, M$ and $N$ be as in Corollary 3.6. Assume that the intersection of all conjugates of $M$ together with all conjugates of $N$ is nontrivial. Then either $M=N=G$, or $M=N$ is an infinite cyclic normal subgroup of $G$.

Proof By Proposition 3.7, we have $M=N$. Assume that $G \neq M$. Then $M=N$ is infinite cyclic by Corollary 3.6. If $M$ is not normal, then there exists $g \in G$ such that $g M g^{-1} \cap M$ is a proper subgroup of $M$. Hence $\bigcap_{i=0}^{\infty} g^{i} M g^{-i}$ must be trivial. This completes the proof of the proposition.

We shall need the following theorem due to Karrass and Solitar in the proof of Theorem 3.10 below.

Theorem 3.9 [19, page 219] Let $G$ be a one-relator group having a (nontrivial) finitely presented normal subgroup $H$ of infinite index. Then $G$ is torsion-free and has two generators. Further, $G$ is an infinite cyclic or infinite dihedral extension of a finitely generated free group $N(\subset G)$ satisfying the following:

- $H \subset N$ if $H$ is not cyclic.

- $H \cap N$ is trivial if $H$ is cyclic.

In either case, there is a finite index subgroup $G_{1}$ of $G$ and a finitely generated free group $F$ fitting in an exact sequence

$$
1 \longrightarrow F \longrightarrow G_{1} \longrightarrow \mathbb{Z} \longrightarrow 1 \text {. }
$$

Theorem 3.10 Let $G=\left\langle x_{1}, \ldots, x_{n} \mid w\right\rangle$ be a one-relator Kähler group. Then either $G$ is virtually a surface group, or there is a finite index subgroup $G_{1}$ of $G$ and a finitely generated free group $F$ fitting in an exact sequence

$$
1 \longrightarrow F \longrightarrow G_{1} \longrightarrow \mathbb{Z} \longrightarrow 1 \text {. }
$$


Proof By Lemma 3.1, the exponent sum of $x_{1}$ in $w$ is zero. Hence from Theorem 3.3 it follows that there exists a finitely generated one-relator group $H$ such that $G$ is an HNN extension $G=H_{* F}$, where $F$ is a nontrivial free group. Also, the associated subgroups $M_{1}$ and $M_{2}$ are Magnus subgroups of $H$.

Let $T$ be the Bass-Serre tree of the splitting of $G$ over $H$. If $T$ is quasi-isometric to $\mathbb{R}$, then there is a finite index subgroup $G_{1}$ of $G$ and a finitely generated free group $F$ fitting in an exact sequence

$$
1 \longrightarrow F \longrightarrow G_{1} \longrightarrow \mathbb{Z} \longrightarrow 1
$$

where $F$ is of finite index in both $M_{1}$ and $M_{2}$; therefore $H$ is free.

Assume that $T$ is not quasi-isometric to $\mathbb{R}$. Then $H$ is a stable cut subgroup in the sense of [11]. This is because $T$ must have infinitely many ends and hence be nonamenable. Hence by [11] (or by Proposition 2.5), there is a surjective homomorphism from $G$ to a surface group $\Gamma_{g}$ of genus $g>0$ with kernel $N \subset H$. If $N$ is finite, then $G$ is virtually a surface group.

Assume that $N$ is infinite. Let $t$ be the stable letter of the HNN extension $G=H_{* F}$. Hence $N \subset H \cap t H t^{-1} \subset M_{1} \cap M_{2}$. Since $N$ is nontrivial, from Proposition 3.8 we conclude that either

(a) $M_{1}=M_{2}=H$, or

(b) $M_{1}=M_{2}$ is an infinite cyclic normal subgroup of $H$.

In case (a), the group $G$ fits in $1 \rightarrow F \rightarrow G \rightarrow \mathbb{Z} \rightarrow 1$, where $F=M_{1}=M_{2}=H$ is free.

In case (b), the subgroup $N \subset M_{1} \cap M_{2}$ must be infinite cyclic, in particular, $G$ admits an infinite cyclic normal subgroup. Hence by Theorem 3.9, there is a finite index subgroup $G_{1}$ of $G$ and a finitely generated free group $F$ fitting in an exact sequence $1 \rightarrow F \rightarrow G_{1} \rightarrow \mathbb{Z} \rightarrow 1$. This proves the theorem.

In the next section we shall rule out the second possibility in Theorem 3.10.

\section{Coherent groups}

\subsection{Torsion-free one-relator Kähler groups}

A finitely presented group $G$ is said to be coherent if every finitely generated subgroup of $G$ is finitely presented. We shall be requiring the following deep theorem due to Feighn and Handel: 
Theorem 4.1 [12] Let $G$ be a finitely presented group admitting a description

$$
1 \longrightarrow F \longrightarrow G \longrightarrow \mathbb{Z} \longrightarrow 1,
$$

where $F$ is finitely generated free. Then $G$ is coherent.

A group is said to be curve-dominating if it possesses a surjective homomorphism onto the fundamental group $\Gamma_{g}$ of a closed Riemann surface of genus $g>1$.

Theorem 4.2 [3, Sections 7J and K] Let $G$ be a curve-dominating Kähler group. Then

$$
\operatorname{dim} H_{1}\left(G^{\prime}, \mathbb{Q}\right)=\infty,
$$

where $G^{\prime}$ denotes the commutator subgroup of $G$. Conversely, if $\operatorname{dim} H_{1}\left(G^{\prime}, \mathbb{Q}\right)=\infty$, then $G$ contains a curve-dominating subgroup of finite index.

Proposition 4.3 Let $G$ be a finitely presented group admitting a description

$$
1 \longrightarrow F \longrightarrow G \longrightarrow \mathbb{Z} \longrightarrow 1
$$

where $F$ is finitely generated free. If $G$ is Kähler, then $G=\mathbb{Z} \oplus \mathbb{Z}$.

Proof Suppose that $G \neq \mathbb{Z} \oplus \mathbb{Z}$. Then $F$ has rank greater than one; the only other case is that of the fundamental group of a Klein bottle, which has first Betti number one and hence it cannot be Kähler. As $G / F$ is abelian, it follows that $G^{\prime} \subset F$ (as before, $G^{\prime}$ is the commutator subgroup of $G$ ). Hence $G^{\prime}$ is a normal subgroup of $F$. Further, since $G$ is nonabelian (as $F$ has rank greater than one), we have $G^{\prime} \neq\{1\}$. As subgroups of free groups are free, it follows that $G^{\prime}$ is a free group.

Since $b_{1}(G)$ is even and $b_{1}(G) \geq 1$, it follows that $G^{\prime} \subset F$ must be of infinite index in $F$. Hence $\operatorname{dim} H_{1}\left(G^{\prime}, \mathbb{Q}\right)=\infty$. So $G$ contains a curve-dominating subgroup of finite index by Theorem 4.2.

By the Siu-Beauville theorem [2, page 2, Theorem 1.5], there exists a holomorphic map

$$
\phi: X \longrightarrow S,
$$

from a compact Kähler manifold to a closed Riemann surface $S$ of genus $g>1$, with connected fibers.

It now follows (see [9, page 283, Lemma 3]) ${ }^{1}$ that there exists a finite-sheeted cover $X_{1}$ of $X$ and a closed Riemann surface $S_{1}$ (possibly different from $S$ ) of genus greater

\footnotetext{
${ }^{1}$ We are grateful to Dieter Kotschick for informing us that we need this refinement to get rid of multiple fibers and for our argument to work.
} 
than one such that $\phi$ lifts to a holomorphic map

$$
\phi_{1}: X_{1} \longrightarrow S_{1},
$$

from $X_{1}$ to $S_{1}$, with connected fibers and no multiple fibers.

As the fibers of $\phi$ are connected and compact and there are no multiple fibers, it follows that the kernel $N$ of $\phi_{1 *}$ is finitely generated. By Theorem 4.1, this group $N$ is finitely presented. Since $N \subset G_{1}$ has infinite index, it must be finitely generated free by Theorem 2.3. Thus $G_{1}$ fits in an exact sequence

$$
1 \longrightarrow F \longrightarrow G_{1} \longrightarrow \pi_{1}\left(S_{1}\right) \longrightarrow 1,
$$

where $F$ is finitely generated free and hence a one dimensional duality group. From Theorem 2.2 it follows that $G_{1}$ has cohomological dimension $1+2=3$. Hence (by Serre's theorem on finite index subgroups) $G$ has cohomological dimension 3 .

Again, since $G$ fits in an exact sequence

$$
1 \longrightarrow F \longrightarrow G \longrightarrow \mathbb{Z} \longrightarrow 1
$$

where $F$ is finitely generated free, it follows from Theorem 2.2 again that $G$ has cohomological dimension $1+1=2$. This is in contradiction with the previous calculation.

Hence $F$ has rank one, and $G=\mathbb{Z} \oplus \mathbb{Z}$.

Combining Theorem 3.10 and Proposition 4.3 we have:

Theorem 4.4 Let $G=\left\langle x_{1}, \ldots, x_{n} \mid w\right\rangle$ be a one-relator Kähler group. Then $G$ is virtually a surface group. Further, if $G$ is torsion-free, then it is isomorphic to a surface group.

Proof The first statement in the theorem is a direct consequence of Theorem 3.10 and Proposition 4.3 because $\mathbb{Z} \oplus \mathbb{Z}$ is a surface group. The last statement follows from the fact that a torsion-free group that is virtually a surface group is actually a surface group.

The case where $G$ has torsion will be dealt in Section 5.1.

The proof of Proposition 4.3 actually gives the following: 
Corollary 4.5 Let $G_{0}$ be a coherent Kähler group of rational cohomological dimension two such that a finite index subgroup $G$ of $G_{0}$ admits a surjective homomorphism

$$
\phi_{*}: G \longrightarrow \pi_{1}(S),
$$

where $S$ is a closed oriented 2-dimensional surface of genus $g>1$. Then $G_{0}$ is virtually a (real two-dimensional) surface group.

Proof As in the proof of Proposition 4.3, there exists a holomorphic map $\psi: X \rightarrow T$ from a compact Kähler manifold to a closed Riemann surface $T$ of genus $g>1$ with connected fibers inducing a surjection $\psi_{*}$ of $G$ onto $\pi_{1}(T)$; we note that $T$ need not be the same as $S$ but will, in general, be a finite-sheeted cover of $S$. Again, the kernel $N$ of $\psi_{*}$ is finitely presented. If $N$ is infinite, then the rational cohomological dimension must be 3 by Theorem 2.2, contradicting the hypothesis. Hence $N$ must be finite, and the result follows.

The virtual first Betti number of a manifold $M$ is defined to be

$$
v b_{1}(M):=\sup \left\{b_{1}(N) \mid N \text { is a finite-sheeted cover of } M\right\} .
$$

Similarly, for a group $G$, define the virtual first Betti number $v b_{1}(G)$ to be

$$
v b_{1}(G):=\sup \left\{b_{1}(H) \mid H \text { is a finite-index subgroup of } G\right\} .
$$

To generalize Proposition 4.3 further, we shall require certain properties of the Albanese map. The following lemma will be used to strengthen Proposition 4.3 to Corollary 4.7 below. This is a special case of a theorem of Catanese, [2, page 23, Proposition 2.4], and a simple self-contained proof may be found in [21, Section 2] by taking $M$ (in the statement of Lemma 4.6 below) to be a cover with positive $b_{1}$.

Lemma 4.6 Let $M_{0}$ be a compact Kähler manifold, with $\pi_{1}\left(M_{0}\right)=G$, such that the real cohomological dimension $\operatorname{cd}_{\mathbb{R}}(G)<4$, and $v b_{1}\left(M_{0}\right)>0$. Then there exists a finite-sheeted cover $M$ of $M_{0}$ such that the image of the Albanese map $F$ for $M$ is a smooth algebraic curve $S$ of genus greater than zero.

Combining Lemma 4.6 with the proof of Proposition 4.3 we get the following.

Corollary 4.7 Let $G$ be a coherent Kähler group of rational cohomological dimension two and $v b_{1}(G)>0$. Then $G$ is virtually a (real two-dimensional) surface group. 
Proof By Lemma 4.6, we obtain a holomorphic map $F: M \rightarrow S$ onto a Riemann surface $S$ of genus greater than zero, where $\pi_{1}(M)$ is a finite index subgroup of $G$. By the Stein factorization theorem, we can assume (by passing to a further finite-sheeted cover if necessary) that the fibers of $F$ are connected. As in the proof of Proposition 4.3, we can further assume that $F$ has no multiple fibers. Hence we have an exact sequence

$$
1 \longrightarrow N \longrightarrow \pi_{1}(M) \longrightarrow \pi_{1}(S) \longrightarrow 1
$$

with $N$ finitely generated, forcing $N$ to be finitely generated free as in Proposition 4.3. This forces the rational cohomological dimension of $\pi_{1}(M)$ to be three, contradicting the hypothesis.

\section{Orbifold groups}

\subsection{One-relator groups with torsion}

Throughout this subsection, $G=\left\langle x_{1}, \ldots, x_{k} \mid w^{n}\right\rangle$ will be a one-relator group, where $k>1, n \geq 1$, and $w$ is cyclically reduced and not a proper power.

If $n=1$, then it is known that $G$ is torsion-free [23, page 266, Theorem 4.12]. Therefore, in view of Theorem 4.4, we are allowed to assume that $n>1$.

Fischer, Karrass and Solitar prove the following:

Proposition 5.1 [13, Theorem 1] Let $G=\left\langle x_{1}, \ldots, x_{k} \mid w^{n}\right\rangle$ be a one-relator group with $n>1$. Then the following statements hold:

(1) Every torsion element in $G$ is conjugate to a power of $w$.

(2) The subgroup generated by torsion elements in $G$ is the free product of the conjugates of $w$.

Murasugi has described in detail the centers of one-relator groups.

Theorem 5.2 [26, Theorems 1 and 2] Let $G=\left\langle x_{1}, \ldots, x_{k} \mid w^{n}\right\rangle$ be a one-relator group with $n \geq 1$. If $k \geq 3$, then the center $Z(G)$ of $G$ is trivial. If $G$ is nonabelian, $k=2$ and $Z(G)$ is nontrivial, then $Z(G)$ is infinite cyclic.

Proposition 5.3 Let

$$
G=\left\langle x_{1}, \ldots, x_{k} \mid w^{n}\right\rangle, \quad n>1,
$$

be a one-relator Kähler group that contains a finite index subgroup isomorphic to the fundamental group of a closed orientable surface $\Sigma$ of genus greater than one. Then $G$ 
is isomorphic to the fundamental group of a hyperbolic (real) two-dimensional compact orbifold $V$ with exactly one cone-point. Further, the underlying manifold of $V$ is orientable.

Proof The group $G$ is clearly a (Gromov) hyperbolic with boundary homeomorphic to the circle $S^{1}$ [17]. The group $G$ acts naturally on the boundary $S^{1}$. Let $N$ be the kernel of the action, meaning

$$
N=\left\{z \in G \mid z(x)=x \forall x \in S^{1}\right\} .
$$

Then $G$ is isomorphic to $N \times(G / N)$, where $G / N$ acts effectively on $S^{1}$ as a convergence group. By a deep theorem of Casson-Jungreis [8], and (independently) Gabai [14], it follows that $G / N$ is the fundamental group of a compact hyperbolic orbifold $V$ of dimension two.

By Proposition 5.1, the kernel $N$ must be cyclic. Hence $N$ is contained in the center of $G$. Therefore, by Theorem 5.2, the group $N$ is trivial. Finally, since all torsion elements of $G$ are conjugate, the orbifold $V$ must have a unique cone point. In fact, by the explicit description of presentations of orbifold groups given in [27], either

(a) $G=\left\langle a_{1}, b_{1}, \ldots, a_{g}, b_{g} \mid w^{n}\right\rangle$, where $w=\prod_{i=1}^{g}\left[a_{i}, b_{i}\right]$, and the underlying manifold is orientable, or

(b) $G=\left\langle a_{1}, \ldots, a_{g} \mid w^{n}\right\rangle$, where $w=\prod_{i=1}^{g} a_{i}^{2}$, and the underlying manifold is nonorientable.

Case (b) is ruled out by Lemma 3.1. This completes the proof of the proposition.

Proposition 5.4 Let $G=\left\langle x_{1}, \ldots, x_{k} \mid w^{n}\right\rangle$ be a one-relator Kähler group such that $G$ contains a finite index subgroup isomorphic to $\mathbb{Z} \oplus \mathbb{Z}$. Then $G$ is isomorphic to $\mathbb{Z} \oplus \mathbb{Z}$.

Proof By Lemma 3.1, the integer $k$ is even and $G$ admits a surjection onto the fundamental group of a closed orientable surface $S$ of genus $k / 2$. Since $G$ contains a finite index subgroup isomorphic to $\mathbb{Z} \oplus \mathbb{Z}$, it follows that $\mathbb{Z} \oplus \mathbb{Z}$ admits a surjection onto the fundamental group of a finite sheeted cover of $S$. Hence $k=2$. Consequently, $G$ admits a right-split exact sequence

$$
1 \longrightarrow N \longrightarrow G \longrightarrow Q(=\mathbb{Z} \oplus \mathbb{Z}) \longrightarrow 1 .
$$

Let $H \subset G$ be a subgroup mapping isomorphically onto $Q$.

As in the proof of Proposition 5.3, the group $N$ must be finite cyclic by Proposition 5.1. It follows that there exists a positive integer $n$ such that $h^{n}$ is in the center of $G$ for 
each $h \in H$. In particular, the center of $G$ contains $\mathbb{Z} \oplus \mathbb{Z}$. By Theorem 5.2, the group $G$ must be abelian.

By Lemma 3.1 again, the abelianization of $G$ is exactly $\mathbb{Z} \oplus \mathbb{Z}$. Since $G$ is itself abelian, the proposition follows.

\subsection{The main theorem}

Combining Theorem 4.4, Proposition 5.3 and Proposition 5.4, we have:

Theorem 5.5 Let $G=\left\langle x_{1}, \ldots, x_{k} \mid w\right\rangle$ be an infinite one-relator Kähler group. Then

$$
G=\left\langle a_{1}, b_{1}, \ldots, a_{g}, b_{g} \mid\left(\prod_{i=1}^{g}\left[a_{i}, b_{i}\right]\right)^{n}\right\rangle
$$

for some $g \geq 1$ and $n \geq 1$.

\subsection{Examples}

Take positive integers $g$ and $n$. We will show that the group

$$
G:=\left\langle a_{1}, b_{1}, \ldots, a_{g}, b_{g} \mid\left(\prod_{i=1}^{g}\left[a_{i}, b_{i}\right]\right)^{n}\right\rangle
$$

is the fundamental group of a smooth complex projective variety. We assume that $n>1$, because $G$ for $n=1$ is a surface group.

Let $f: X \rightarrow S$ be a smooth projective elliptic surface satisfying the following conditions:

- The genus of the Riemann surface $S$ is $g$.

- There is a point $x_{0} \in S$ such that the gcd of the multiplicities of the irreducible components of the fiber over $x_{0}$ is $n$.

- The reduced fiber over some point (it can be $x_{0}$ ) is singular.

- For each point $x \in S \backslash\left\{x_{0}\right\}$, the gcd of the multiplicities of the irreducible components of the fiber over $x$ is 1 .

The fundamental group of $X$ is the group $G$ defined above. To see this, consider the short exact sequence in the bottom half of [28, page 600]. The group $\mathcal{H}_{f}$ in this exact sequence coincides with $G$ [28, page 601, Lemma 2]. The group $\mathcal{V}_{f}$ in the exact sequence vanishes [28, page 614, Theorem 4]. Therefore, from this short exact sequence we conclude that $\pi_{1}(X)=G$. 
Acknowledgments Different parts of this work were done while the authors were vising Harish-Chandra Research Institute, Allahabad, and Institute of Mathematical Sciences, Chennai, and during a visit of the second author to Tata Institute of Fundamental Research, Mumbai. We thank these institutions for their hospitality. We would like to thank Dieter Kotschick for pointing out a small gap in an earlier version of Proposition 4.3 and for pointing out the proof in Section 2 of [21] to us. Recently Kotschick [20] has found a new proof of Theorem 5.5 by techniques involving $l^{2}$ cohomology.

Research of the second author is partly supported by CEFIPRA Indo-French research grant number 4301-1.

\section{References}

[1] J Amorós, On the Malcev completion of Kähler groups, Comment. Math. Helv. 71 (1996) 192-212 MR1396672

[2] J Amorós, M Burger, K Corlette, D Kotschick, D Toledo, Fundamental groups of compact Kähler manifolds, Mathematical Surveys and Monographs 44, American Mathematical Society (1996) MR1379330

[3] D Arapura, Fundamental groups of smooth projective varieties, from: "Current topics in complex algebraic geometry", (H Clemens, J Kollár, editors), Math. Sci. Res. Inst. Publ. 28, Cambridge Univ. Press (1995) 1-16 MR1397055

[4] R Bieri, Normal subgroups in duality groups and in groups of cohomological dimension 2, J. Pure Appl. Algebra 7 (1976) 35-51 MR0390078

[5] R Bieri, B Eckmann, Groups with homological duality generalizing Poincaré duality, Invent. Math. 20 (1973) 103-124 MR0340449

[6] I Biswas, M Mj, H Seshadri, Three manifold groups, Kähler groups and complex surfaces, to appear in Commun. Contemp. Math. arXiv:1101.1162

[7] K S Brown, Cohomology of groups, Graduate Texts in Mathematics 87, Springer, New York (1982) MR672956

[8] A Casson, D Jungreis, Convergence groups and Seifert fibered 3-manifolds, Invent. Math. 118 (1994) 441-456 MR1296353

[9] F Catanese, J Keum, K Oguiso, Some remarks on the universal cover of an open K3 surface, Math. Ann. 325 (2003) 279-286 MR1962049

[10] D J Collins, Intersections of conjugates of Magnus subgroups of one-relator groups, from: "The Zieschang Gedenkschrift", (M Boileau, M Scharlemann, R Weidmann, editors), Geom. Topol. Monogr. 14 (2008) 135-171 MR2484702 
[11] T Delzant, M Gromov, Cuts in Kähler groups, from: "Infinite groups: geometric, combinatorial and dynamical aspects", (L Bartholdi, T Ceccherini-Silberstein, T Smirnova-Nagnibeda, A Zuk, editors), Progr. Math. 248, Birkhäuser, Basel (2005) 31-55 MR2195452

[12] M Feighn, M Handel, Mapping tori of free group automorphisms are coherent, Ann. of Math. 149 (1999) 1061-1077 MR1709311

[13] J Fischer, A Karrass, D Solitar, On one-relator groups having elements of finite order, Proc. Amer. Math. Soc. 33 (1972) 297-301 MR0311780

[14] D Gabai, Convergence groups are Fuchsian groups, Ann. of Math. 136 (1992) 447-510 MR1189862

[15] R Gitik, M Mitra, E Rips, M Sageev, Widths of subgroups, Trans. Amer. Math. Soc. 350 (1998) 321-329 MR1389776

[16] M Green, R Lazarsfeld, Higher obstructions to deforming cohomology groups of line bundles, J. Amer. Math. Soc. 4 (1991) 87-103 MR1076513

[17] M Gromov, Hyperbolic groups, from: "Essays in group theory", (S M Gersten, editor), Math. Sci. Res. Inst. Publ. 8, Springer, New York (1987) 75-263 MR919829

[18] M Gromov, Sur le groupe fondamental d'une variété kählérienne, C. R. Acad. Sci. Paris Sér. I Math. 308 (1989) 67-70 MR983460

[19] A Karrass, D Solitar, One relator groups having a finitely presented normal subgroup, Proc. Amer. Math. Soc. 69 (1978) 219-222 MR0466323

[20] D Kotschick, The deficiencies of Kähler groups arXiv:1204:6278

[21] D Kotschick, Three-manifolds and Kähler groups, to appear in Ann. Inst. Fourier arXiv: 1011.4084

[22] R C Lyndon, P E Schupp, Combinatorial group theory, Ergeb. Math. Grenzgeb. 89, Springer, Berlin (1977) MR0577064

[23] W Magnus, A Karrass, D Solitar, Combinatorial group theory: Presentations of groups in terms of generators and relations, Interscience, New York (1966) MR0207802

[24] J McCool, P E Schupp, On one relator groups and HNN extensions, J. Austral. Math. Soc. 16 (1973) 249-256 MR0338186

[25] D I Moldavanskiü, Certain subgroups of groups with one defining relation, Sibirsk. Mat. Ž. 8 (1967) 1370-1384 MR0220810

[26] K Murasugi, The center of a group with a single defining relation, Math. Ann. 155 (1964) 246-251 MR0163945

[27] P Scott, The geometries of 3-manifolds, Bull. London Math. Soc. 15 (1983) 401-487 MR705527 
[28] G Xiao, $\pi_{1}$ of elliptic and hyperelliptic surfaces, Internat. J. Math. 2 (1991) 599-615 MR1124285

School of Mathematics, Tata Institute of Fundamental Research Homi Bhabha Road, Bombay 400005, India

Department of Mathematics, RKM Vivekananda University P.O. Belur Math, Dt Howrah, Howrah 711202, India

indranil@math.tifr.res.in, mahan@rkmvu.ac.in

http://maths.rkmvu.ac.in/ mahan/

Proposed: Benson Farb

Received: 3 February 2012

Seconded: Ronald Stern, David Gabai

Revised: 30 April 2012 